\title{
Motion Artifact Correction of Multi-Photon Imaging of Awake Mice Models Using Speed Embedded HMM
}

\author{
Taoyi Chen ${ }^{1,2}$, Zhong Xue ${ }^{1}$, Changhong Wang ${ }^{2}$, Zhenshen $\mathrm{Qu}^{2}$, Kelvin K. Wong ${ }^{1}$, \\ and Stephen T.C. Wong ${ }^{1}$
}

\author{
${ }^{1}$ Center for Bioengineering and Informatics, Methodist Hospital Research Institute and \\ Department of Radiology, The Methodist Hospital, Weill Cornell Medical College, Houston, TX \\ ${ }^{2}$ Department of Control Science and Engineering, Harbin Institute of Technology, China \\ zxue@tmhs.org
}

\begin{abstract}
Multi-photon fluorescence microscopy (MFM) captures highresolution anatomical and functional fluorescence image sequences and can be used for the intact brain imaging of small animals. Recently, it has been extended from imaging anesthetized and head-stabilized animals to awake and head-restrained ones for in vivo neurological study. In these applications, motion correction is an important pre-processing step since brain pulsation and tiny body movement can cause motion artifacts and prevent stable serial image acquisition at such a high spatial resolution. This paper proposes a speed embedded hidden Markov model (SEHMM) for motion correction in MFM imaging of awake head-restrained mice. The algorithm extends the traditional HMM method by embedding a motion prediction model to better estimate the state transition probability. SEHMM is a line-by-line motion correction algorithm, which is implemented within the in-focal-plane 2-D videos and can operate directly on the motion-distorted imaging data without external signal measurements such as the movement, heartbeat, respiration, or muscular tension. In experiments, we demonstrat that SEHMM is more accurate than traditional HMM using both simulated and real MFM image sequences.
\end{abstract}

\section{Introduction}

Mammalian brain microscopy plays an important role in studying the electrical and biochemical processes in neurons. Previously, CCD imaging had been used to study mouse brain [1], in which a single fiber-optic bundle is placed in direct contact in the barrel cortex of an awake mouse to image cortical activities in conjunction with voltage sensitive dye. Since fiber-optic bundle have intrinsic low spatial resolution limited by the size of the fiber core, such kind of imaging system does not reach cellularresolution but allows researchers to obtain cortical activation map with high temporal resolution. Recently, multi-photon fluorescence microscopy (MFM) imaging has been used to capture neural images from anesthetized mice at higher spatial and temporal resolution [2-5]. In [2] and [5], $\mathrm{Ca}^{2+}$ transients were quantified using MFM in the mice models of Alzheimer's disease (AD). Unfortunately, animals under anesthesia cannot demonstrate the neural dynamics sufficiently, and overall brain activities are suppressed. Recently, more MFM imaging studies of awake mice were carried out 
[6-9], and two-photon microscope (TPM), a special variant of MFM, was demonstrated as a superior alternative due to its deeper tissue penetration, efficient light detection, and reduced phototoxicity [3].

Head-restrained TPM [8,9] uses a standard microscope system to visualize simultaneous $\mathrm{Ca}^{2+}$ dynamics of a large number of individual cells, while the head-restrained mouse is free to walk or run on a stationary exercise ball [10]. Because of the mice's random motion, the relative motion between the microscope and the brain leads to line shifting artifacts, which affects image reconstruction and quantification of the functional dynamics. Thus a motion correction algorithm is needed for quantifying brain motion and aiding in stabilizing cell displacements within a region of interest (ROI) in order to reduce the number of brain-motion-related fluorescence transients.

In the literature, two methods were proposed for the motion correction of TPM in awake mice [8, 9]. Greenberg and Kerr [9] proposed a method based on the LucasKanade algorithm $[11,12]$ to estimate the offset sequences by minimizing the squared differences between a reference frame and the subsequent frames using gradient descent method. In [8], an HMM-based method was proposed to calculate the optimal offset using a probabilistic framework. Compared to [11, 12], HMM algorithm generates more temporally stable results due to the implicit modeling of longitudinal state transition. Basically, the HMM algorithm assumes that the motion of the current line to the next line remains the same or is most likely not changing. A standard exponential model (or distribution) is used to describe this assumption. Essentially, this assumption is only realistic when the mice are still or relatively still (the relative position for scanning beam and mice's local neural region of interest stays constant), but this situation seems not very common because mice's motion is disordered and random and includes sudden changes of speed all the time during the procedure. Thus, in HMM, although the state transition model works effectively for the resting stage, it might fail and give a wrong estimation during the running stage.

In this paper, we extend the HMM by incorporating an estimated speed into the state transition model for more accurate estimation, called speed embedded HMM (SEHMM) algorithm. The major contributions for SEHMM are as follows. (1) Initial motion estimation is achieved by using a simple line-by-line searching method; (2) a grouping algorithm is used to divide the whole imaging period into resting and running stages for speed estimation; (3) SEHMM is then adopted for motion correction. In experiments, a quantitative validation was performed to compare HMM with SEHMM based on both simulated data and real data. First, simulated image sequences were generated to mimic various real motion situations, and we applied different dynamic amplitudes to make the validation more realistic and reasonable. For real data, we showed the comparative results to demonstrate the performance of SEHMM. The results illustrated that SEHMM can achieve higher estimation accuracy of image alignment as compared with HMM, especially in the running stages of the image sequences.

\section{Method}

\subsection{Problem Formulation}

A two-photon microscope captures a series of images by passing a focus of laser excitation repeatedly over a rectangular or square region of fluorescently labeled 
tissue and collecting the resulting photons via a photon multiplier tube (PMT) [13]. Although the mice's motion is in 3-D, Z-axis motion shift is less than $1 \mu m$ for a scanning speed of $2 \mathrm{~ms} / \mathrm{line}$, much lower than the motion along the $\mathrm{X}$ (medial-lateral direction along the raster scan line) and $\mathrm{Y}$ (rostral-caudal direction across the raster scan line) directions. Therefore, our task is to estimate the motion in $\mathrm{X}$ - and $\mathrm{Y}$-directions. Due to the mice's motion during the raster scan progression, the relative motion can be written as,

$$
\left\{\begin{array}{l}
X_{i}^{\prime k}=X_{i}^{k}+\delta_{x}^{k} \\
Y_{i}^{\prime k}=Y_{i}^{k}+\delta_{y}^{k}
\end{array},\right.
$$

where $X_{i}^{k}(t)=\{t /(\tau / N)\} \cdot N$ and $X_{i}^{k}(t)=[t /(\tau / N)]$ give the actual location of an object point, and $\left(\delta_{x}^{k}, \delta_{y}^{k}\right)$ is its offset due to motion. $N \times N$ is the size of each frame. [·] represents the integer operation, and $\{\cdot\}$ denotes the fractional operation. $\tau$ is the scanning time for a frame. It can be seen that the laser moves in a zigzag pattern in Xdirection and a step function pattern in Y-direction. Our goal is to estimate the offsets $\left(\delta_{x}^{k}, \delta_{y}^{k}\right)$ from the serial images. Eq. (1) assumes each line has the same relative displacement, so we can choose a line-by-line motion correction algorithm to solve this problem. The reason is that the shifts for all the pixels within a line do not get beyond one-pixel in Y-direction and are very tiny in $\mathrm{X}$ direction. Although pixel-by-pixel correction can yield more accurate results, one has to trade off between computational speed and the gain in accuracy.

\subsection{The Speed Embedded HMM (SEHMM) Algorithm}

The speed embedded HMM (SEHMM) algorithm is an extension of the traditional HMM method by using a motion prediction model with HMM to better estimate the state transition probability. Denoting the displacement state for line $k$ as $\left(\delta_{x}, \delta_{y}\right)$, the state observation probability is represented as $\pi_{k}^{\delta_{x}, \delta_{y}}$, and the state transition probability is denoted as $T\left[\left(\delta_{x}^{k-1}, \delta_{y}^{k-1}\right) \rightarrow\left(\delta_{x}^{k}, \delta_{y}^{k}\right)\right]$. The idea of SEHMM is that the transition probability of the displacement state is estimated based on the estimated moving speed. Thus, SEHMM can match motion more accurately not only during the resting stage but also during the moving stage.

In SEHMM, first, the transition probability is defined as,

$$
T\left(\delta_{x}^{k-1}, \delta_{y}^{k-1}\right) \rightarrow\left(\delta_{x}^{k}, \delta_{y}^{k}\right)=\frac{1}{2 \pi \lambda} e^{-r / \lambda},
$$

where $r$ is defined as:

$$
r=\sqrt{\left(\delta_{x}^{k}-\left(\delta_{x}^{k-1}+v_{x}^{k-1, k} \tau_{\text {line }}\right)\right)^{2}+\left(\delta_{y}^{k}-\left(\delta_{y}^{k-1}+v_{y}^{k-1, k} \tau_{\text {line }}\right)\right)^{2}},
$$

where $v_{x}^{k-1, k}$ and $v_{y}^{k-1, k}$ are the estimated speed of the motion from line $k-1$ to $k$ for $\mathrm{X}$ - and $\mathrm{Y}$-directions, respectively. $\tau_{\text {line }}$ is the scanning time for a line. It can be seen that using Eq. (3), if the moving speed is estimated at line $k-1$, the offset at line $k$ can be estimated. Therefore, in SEHMM, we no longer assume that the state transition probability is the highest when the mouse does not move. On the contrary, this 
probability gets its peak at a linearly estimated offset value. Since the goal for motion correction is to estimate $\delta_{x}^{k}, \delta_{y}^{k}$ for each line $k$ from a given reference frame $R$ and the current image $I$, we implement it by maximizing a posteriori,

$$
P=\left(\delta_{x}^{k}, \delta_{y}^{k} I_{i}^{k}, R\right)=P\left(I_{i}^{k} \mid R\left(X_{i}^{\prime k}, Y_{i}^{\prime k}\right)\right) \cdot P\left(\delta_{x}^{k}, \delta_{y}^{k} \mid \delta_{x}^{k-1}, \delta_{y}^{k-1}\right)=\pi_{k}^{\delta_{x}, \delta_{y}} \cdot T\left(\delta_{x}^{k-1}, \delta_{y}^{k-1}\right) \rightarrow\left(\delta_{x}^{k}, \delta_{y}^{k}\right) .
$$

Expressing $P$ as a logarithm probability, one gets,

$$
\ln (P)=\ln \left(\pi_{k}^{\delta_{x}, \delta_{y}}\right)+\ln (T) .
$$

The first term is the state observation probability $\pi_{k}^{\delta_{x}, \delta_{y}}$ and reflects the goodness of matching between a line in the current frame $I$ and the corresponding line in the reference frame $R$. Denoting the intensity of the $i$ th pixel in the $k$ th line of the current frame as $I_{i}^{k}$, and that of the corresponding pixel in the reference frame as $\left(x_{i}^{k}, y_{i}^{\prime k}\right)$, the observation probability can be modeled as a discrete Poisson distribution explicitly, $\pi_{k, i}^{\delta_{\delta}, \delta_{y}}=(\gamma R)^{\gamma I} \cdot \mathrm{e}^{-\gamma R} /(\gamma I)$ !, where $\gamma$ is the calibration factor of the photon number, which is an inherent factor in an imaging system. Taking the logarithm transformation of $\pi_{k, i}^{\delta_{r}, \delta_{y}}$ we get,

$$
\ln \left(\pi_{k, i}^{\delta_{x}, \delta_{y}}\right)=\gamma I \ln (\gamma)+\gamma I \ln (R)-\ln ((\gamma I) !)-\gamma R,
$$

where $\gamma$ and $I_{i}^{k}$ are independent of the changing offsets, and $R$ is indeed a function of the offsets. Thus the equation can be simplified as,

$$
\ln \left(\pi_{k, i}^{\delta_{x}, \delta_{y}}\right) \propto \gamma(I \ln (R)-R) .
$$

Then, the observation probabilities for all the pixels within line $k$ can be calculated by,

$$
\ln \left(\pi_{k}^{\delta_{k}, \delta_{y}}\right)=\sum_{i=1}^{N} \ln \left(\pi_{k, i}^{\delta_{,}, \delta_{y}}\right) .
$$

To illustrate how this new formulation works, Fig. 1 shows the state transition probabilities for HMM (Fig. 1(a)) and SEHMM (Fig. 1(b)) models, respectively. The center of each distribution plot is set to $\left(\delta_{x}^{k-1}, \delta_{y}^{k-1}\right)$, and it can be seen that the peak of the state transition probability is right in the middle for Fig. 1 (a), and this peak can be shifted in Fig. 1 (b) according to the motion estimation. A shifting of the peak of the probability function indicates that a motion is assumed from one line to another. This compensation for adding the estimated speed value from the current offset to the following offset can remarkably improve the estimation accuracy because it satisfies the prediction requirement in all the time points but not only in the still time points.

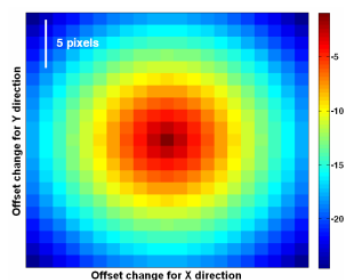

(a)

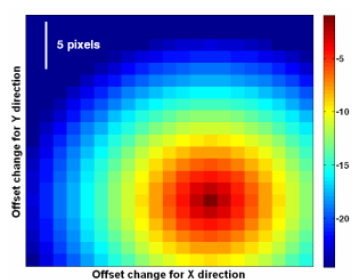

(b)

Fig. 1. The state transition probability functions without (a) and with (b) motion estimation 
Once the state observation probability $\pi_{k}^{\delta_{x}, \delta_{y}}$ and the state transition probability $T\left[\left(\delta_{x}^{k-1}, \delta_{y}^{k-1}\right) \rightarrow\left(\delta_{x}^{k}, \delta_{y}^{k}\right)\right]$ are defined, the best displacement signal can be calculated through maximizing Eq. (5), by following two iterative steps: first, the Expectation Maximization (EM) algorithm [14] can be used to calculate the best $\lambda$ that leads to the highest probability for Eq. (5). Our algorithm for doing this involves systematically scanning values of $\lambda$ in a prescribed range. We scan uniformly in the logarithm space to get constant percentage sampling. For each value of $\lambda$, we run the SEHMM procedure to find the most optimal offset sequence and calculate its total probability. The value of $\lambda$ we will choose is the one with the most probable offset sequence. Then, we can adopt the Viterbi algorithm to find the most likely sequence of the hidden states (offsets). This can be accomplished in two steps. First, we determine the most probable offset sequence for every state at time (line) $k$ from any of the states at time (line) $k-1$ by marching forward through the time domain. Then, we can backtrack along the path of the most probable offset sequence to record the results of optimal offset sequence. In the implementation, a line-by-line search is first used to estimate the initial offset, and the speed of the motion can be estimated by applying the smoothness filter temporally on the estimated offsets before running SEHMM.

\section{Results}

\subsection{Results on Simulated Datasets}

Simulated serial images are used to quantitatively validate the SEHMM algorithm by comparing with the HMM method [8]. Temporal displacements are first generated to transfer a real 2D image (see Fig. 2(left)) to produce the longitudinal image sequences. The displacement signal can be generated through a pixel-by-pixel basis using a first-order differential equation with a characteristic time constant driven by a combination of the random and deterministic processes to simulate the effect of acquiring laser-scanning data from a moving sample:

$$
\left\{\begin{array}{l}
\vec{\phi}_{x}^{t+1}=\left(1-\frac{1}{\tau}\right) \vec{\phi}_{x}^{t}+\frac{1}{\tau}\left(\vec{\zeta}_{\sigma_{x}}+\vec{D}_{x}^{t}\right) \\
\vec{\phi}_{y}^{t+1}=\left(1-\frac{1}{\tau}\right) \vec{\phi}_{y}^{t}+\frac{1}{\tau}\left(\vec{\zeta}_{\sigma_{y}}+\vec{D}_{y}^{t}\right)
\end{array},\right.
$$

where $\bar{\phi}_{x}^{t}$ and $\bar{\phi}_{y}^{t}$ denote the simulated offsets for $\mathrm{X}$ - and Y-directions, respectively, and $t$ expresses the time-point, $t=(k-1) \cdot N+i . \tau$ is set to 500. $\sigma_{x}$ and $\sigma_{y}$ $\left(\sigma_{x}=4, \sigma_{y}=40\right)$ are the standard deviations of the Gaussian random variables $\vec{\zeta}_{\sigma_{x}}$ and $\bar{\zeta}_{\sigma_{v}} . \vec{D}_{x}^{t}$ and $\vec{D}_{y}^{t}$ are the step functions at the time $t$. Then, the Poisson noise is added on a pixel-by-pixel basis to simulate the photon counting statistics because the values during scanning are Poisson distributed in terms of photon numbers but not in units of pixel intensity. $\sigma_{x}, \sigma_{y}$ and $\tau$ control the amplitudes of temporal dynamic displacements. One simulated dataset (T1) was generated with these default parameters. In addition, we also changed the values of $\sigma_{x}, \sigma_{y}$ and $\tau$ and kept the other parameters the same, and the second dataset (T2) was simulated by setting $\sigma_{x}=10, \sigma_{y}=30$ and 

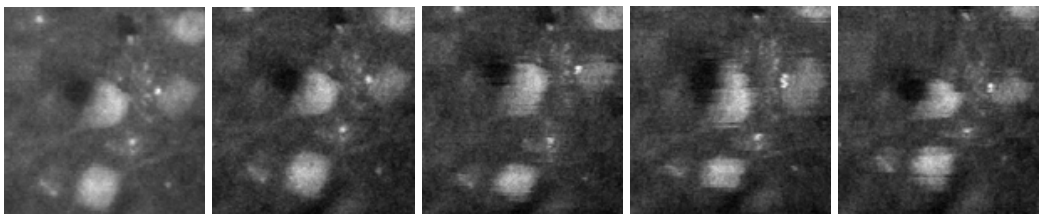

Fig. 2. The reference image (left) and some examples of the simulated images

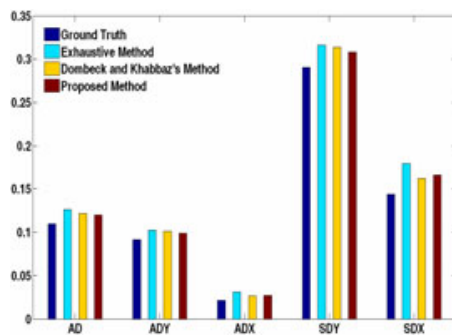

(a)

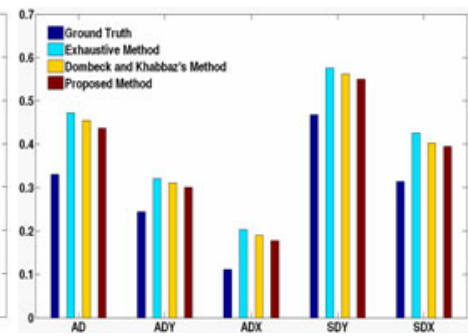

(b)

Fig. 3. Quantitative comparisons for four simulated datasets. (a-b) results of datasets $\mathrm{T} 1$ and $\mathrm{T} 2$.

$\tau=200$. The number of frames for T1 and T2 are 65 and 51, respectively. The image size is $129 \times 129$, and resolution is $0.39 \mu \mathrm{m} /$ pixel.

Denoting the results by HMM as $\left(\delta_{x}^{k}, \delta_{y}^{k}\right)$, and those of SEHMM as $\left(\delta_{x}^{k}, \delta_{y}^{\prime k}\right)$, the simulated offsets as $\left(\phi_{x}^{t}, \phi_{y}^{t}\right)$, and the exhaustive search results as $\left(\hat{\delta}_{x}^{k}, \hat{\delta}_{y}^{k}\right)$, we compared their motion correction accuracy. As all the three methods tested are line-by-line motion correction and the ground truth is stored in pixel resolution, we take the mean value of all ground truth offsets within each line and then round the mean value to the nearest integral, i.e., $\phi_{x}^{t}, \phi_{y}^{t} \rightarrow \phi_{x}^{k}, \phi_{y}^{k}$. The difference between these two data was also compared, which indicates how accurate we can achieve using line-by-line based methods. We used the following five performance measures: average distance $(\mathrm{AD})$, average distance for $\mathrm{Y}$-direction (ADY), average distance for X-direction (ADX), standard deviation for Y-direction (SDY) and standard deviation for X-direction (SDX), to evaluate the performance of the algorithms. Fig. 3 shows the comparison results between the four line-by-line results and the ground truth for data T1 and T2, respectively. The blue bars show differences between the rounded line-by-line ground truth and the simulated pixel-by-pixel ground truth, and other bars show the results of the three methods. It can be seen that for the performance measures, SEHMM yields the smallest errors (some with slightly larger SDX).

We also focused on the Y-direction because the offsets in this direction have relatively large movement than in X-direction. Fig. 4 illustrates the results for simulated dataset T2. In the figure, we show the line-by-line rounded ground truth of and the results of SEHMM. Since these two curves almost overlap each other and it is difficult to visually see the differences, we thus plot the inconsistency between them. The inconsistency is calculated as the number of lines within a frame that the results of SEHMM and HMM are different with the ground truth. It can be seen that during the resting stage, such inconsistency is small, and both SEHMM and HMM yielded good results (about 10 lines are different), and during the running stage, SHEMM can reduce the inconsistency as compared with the results of HMM. 


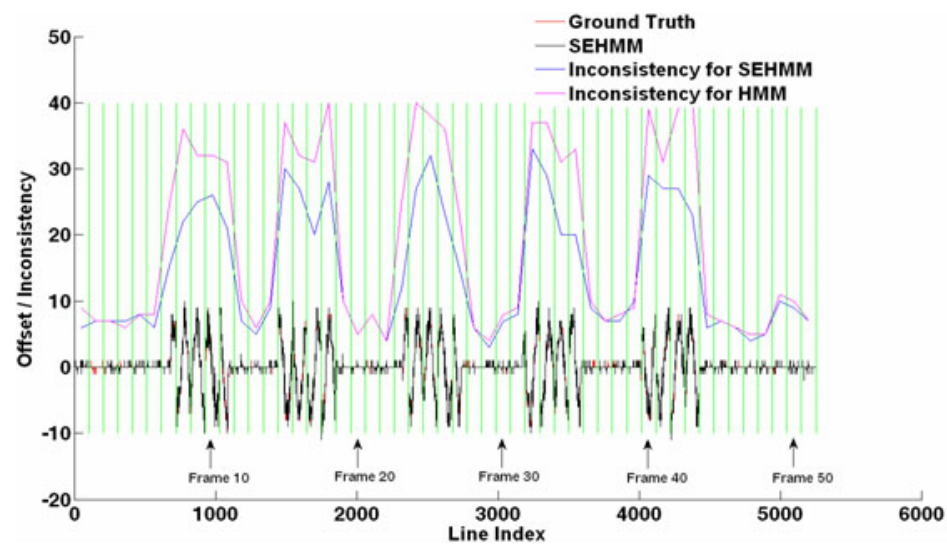

Fig. 4. Comparison results for simulated data T2. Green lines indicate frame separation.

\subsection{Experiments on Real Data}

For real data, we used the mean absolute intensity difference as the measure to show the performance of the methods. The mean absolute intensity difference across longitudinally corresponding pixels reflects the goodness of matching for the image sequences. Table 1 shows the comparison results for data T3 and T4 (provided by Dr. David Tank), respectively, using different methods. The number of frames are 1200 and 200, image size is $128 \times 128$ and 256x256, respectively, and the resolution is 0.39 $\mu \mathrm{m} /$ pixel for both. The improvement can be seen from the comparison results when we adopt the proposed method.

Table 1. Average of absolute intensity differences for real datasets T3 and T4

\begin{tabular}{|c|c|c|c|c|}
\hline Method & Original Data & Exhaustive Method & HMM & SEHMM \\
\hline T3 & 7.05 & 5.89 & 5.48 & 5.03 \\
\hline T4 & 9.54 & 7.74 & 7.15 & 6.56 \\
\hline
\end{tabular}

Regarding computational speed, the exhaustive method is the fastest because limited number of possible motion is considered, and the SEHMM method is slightly more complex than the HMM method because of the embedded exhaustive method in the preprocessing step. However, during the actual motion estimation, HMM and SEHMM have essentially the same speed. Regarding motion correction accuracy, the exhaustive method is not the optimal solution for motion correction because there exist strong line-to-line correlations for offsets of successive lines, and exhaustive method does not consider this point. SEHMM outperforms HMM because the estimated speed of motion is embedded in the model itself.

\section{Conclusion}

We proposed a speed embedded hidden Markov model (SEHMM) for motion correction in MFM imaging. The key idea of SEHMM is that a motion prediction model is utilized to better estimate the state transition probability. Compared to the traditional HMM that assumes no motion always has the highest probability, the proposed 
SEHMM can model the motion more accurately. SEHMM operates directly on the motion-distorted imaging data, without any external signal measurement such as the mice's movements, heartbeat, respiration, or muscular tension. Using simulated and real images, we demonstrated that SEHMM is more accurate than HMM using both simulated and real MFM image sequences.

\section{Acknowledgement}

The authors would like to thank Dr. David Tank from Princeton University for providing the data.

\section{References}

1. Ferezou, I., Bolea, S., Petersen, C.C.: Visualizing the Cortical Representation of Whisker Touch: Voltage-Sensitive Dye Imaging in Freely Moving Mice. Neuron 50, 617-629 (2006)

2. Kuchibhotla, K.V., Lattarulo, C.R., Hyman, B.T., Bacskai, B.J.: Synchronous Hyperactivity and Intercellular Calcium Waves in Astrocytes in Alzheimer Mice. Science 323, 1211 (2009)

3. Denk, W., Strickler, J.H., Webb, W.W.: Two-Photon Laser Scanning Fluorescence Microscopy. Science 248, 73-76 (1990)

4. Denk, W., Svoboda, K.: Photon Upmanship: Techreview Why Multiphoton Imaging Is More Than a Gimmick. Neuron 18, 351-357 (1997)

5. Busche, M.A., Eichhoff, G., Adelsberger, H., Abramowski, D., Wiederhold, K.H., Haass, C., Staufenbiel, M., Konnerth, A., Garaschuk, O.: Clusters of Hyperactive Neurons near Amyloid Plaques in a Mouse Model of Alzheimer's Disease. Science 321, 1686 (2008)

6. Helmchen, F., Fee, M.S., Tank, D.W., Denk, W.: A Miniature Head-Mounted Two-Photon Microscope High-Resolution Brain Imaging in Freely Moving Animals. Neuron 31, 903-912 (2001)

7. Flusberg, B.A., Jung, J.C., Cocker, E.D., Anderson, E.P., Schnitzer, M.J.: In Vivo Brain Imaging Using a Portable 3.9 Gram Two-Photon Fluorescence Microendoscope. Optics letters 30, 2272-2274 (2005)

8. Dombeck, D.A., Khabbaz, A.N., Collman, F., Adelman, T.L., Tank, D.W.: Imaging LargeScale Neural Activity with Cellular Resolution in Awake, Mobile Mice. Neuron 56, 43-57 (2007)

9. Greenberg, D.S., Kerr, J.N.D.: Automated Correction of Fast Motion Artifacts for TwoPhoton Imaging of Awake Animals. Journal of Neuroscience Methods 176, 1-15 (2009)

10. Wilt, B.A., Burns, L.D., Ho, E.T.W., Ghosh, K.K., Mukamel, E.A., Schnitzer, M.J.: Advances in Light Microscopy for Neuroscience. Annual review of neuroscience 32, 435-506 (2009)

11. Lucas, B.D., Kanade, T.: An Iterative Image Registration Technique with an Application to Stereo Vision. In: Proceedings of the 1981 DARPA Image Understanding Workshop, vol. 3, pp. 121-130 (1981)

12. Baker, S., Matthews, I.: Lucas-Kanade 20 Years On: A Unifying Framework. International Journal of Computer Vision 56, 221-255 (2004)

13. Foord, R., Jones, R., Oliver, C.J., Pike, E.R.: The Use of Photomultiplier Tubes for Photon Counting. Applied Optics 8, 1975-1989 (1969)

14. Dempster, A.P., Laird, N.M., Rubin, D.B.: Maximum Likelihood from Incomplete Data Via the Em Algorithm. Journal of the Royal Statistical Society Series B (Methodological), $1-38$ (1977) 